\title{
High-Quality Power Generation Through Distributed Control of a Power Park Microgrid
}

\author{
Milan Prodanović, Member, IEEE, and Timothy C. Green, Senior Member, IEEE
}

\begin{abstract}
Inverters are a necessary interface for several forms of distributed generation (DG) and where they form a microgrid they have the potential to offer high power quality. The challenge is to coordinate the actions of a group of inverters so that they offer the level of power quality known to be possible from fast local control of a single inverter. The case examined here is a power park of several inverter-based DG in relatively close proximity. A basic requirement is that the inverters regulate the grid voltage and share the real and reactive power demands according to their ratings. In small girds with high proportions of nonlinear and unbalanced loads it is also important to actively control the waveform quality in terms of harmonics, transient disturbances, and balance. Further, it is important that these duties are shared equally between the units rather than having one master unit taking the lead in the voltage control function. A constraint faced in designing a sharing system is the limited bandwidth of signal communication even over distances of a few meters. A control method is proposed that separates the control tasks in the frequency domain. Power sharing and voltage regulation are controlled centrally and commands are distributed through a low-bandwidth communication link. Waveform quality functions are controlled in high bandwidth controllers distributed to each local inverter. Experimental tests on a grid of three 10-kVA inverters are used to show that the method fully exploits the inherent fast response of the inverters while also ensuring voltage balance even with extreme load imbalance. It is shown that circulating currents are avoided during steady state and transients.
\end{abstract}

Index Terms-Distributed generation (DG), inverter, microgrid, power quality.

\section{INTRODUCTION}

$\mathbf{M}$ ANY OF the new and renewable forms of energy require an inverter interface in order to export power to a 50or $60-\mathrm{Hz}$ distribution network. The inverter is capable of much more than simple power conversion to ac. Exploiting its fast response in closed-loop can provide rejection of disturbances so that high-quality voltage waveforms are achieved even in the presence of extreme levels of unbalance and harmonically distorted load current. To achieve sufficient control bandwidth to suppress harmonic distortion requires moderately high switching frequency and, thus, the insulated gate bipolar transistor is the device of choice. Because device ratings are limited, the inverter rating must be shared across multiple devices to achieve power ratings in the $100 \mathrm{~kW}$ to $10 \mathrm{MW}$ range. For operational flexibility, it is useful to form parallel connected modules.

Manuscript received December 30, 2004; revised July 20, 2005. Abstract published on the Internet July 14, 2004.

The authors are with the Department of Electrical and Electronic Engineering, Imperial College London, London SW7 2AZ, U.K. (e-mail: proca@ imperial.ac.uk).

Digital Object Identifier 10.1109/TIE.2006.882019
The limitations of a single inverter in terms of component availability, heat dissipation, cost and short-time overload can be eased through a modular approach [1].

The principle of paralleling power electronic modules to form an ac power system emerged first for the purpose of large uninterruptible power supplies (UPS). Several control strategies for parallel operation have been proposed [2]-[4]. These proposals concentrate on single-phase ac systems and three-phase systems have received much less attention in this context. The methods for paralleling three-phase inverters fall into two categories: those that follow the principles established for parallel $\mathrm{dc} / \mathrm{dc}$ converters [5] and those that use the inverter to mimic the operation of a synchronous generator [6], [7]. The difficulty of paralleling voltage source inverters is that circulating currents can flow between units if the output voltages or impedances of the units are mismatched. Since perfect matching is impossible and even small mismatches can cause large currents, a means of ensuring sharing of current is essential. A well established approach is to use nested control loops in which each voltage source inverter is controlled to follow a current reference. These current sources are then used within a voltage control loop working to a reference for the grid voltage. Therefore, although we start with voltage sources and wish to create a bus voltage, we have intermediate current control loops. So long as these current controllers follow their references, current sharing is assured. Poor sharing is sometimes described in terms of the circulating current flowing between units such that their output currents are displaced from the desired value. Accurate sharing is equivalent to eliminating circulating currents.

The connection distance between inverters is an important consideration in systems of parallel modules and not just in terms of the connection impedances but also in terms of how control signals are distributed. In microgrids in remote areas, such as an island with a photovoltaic-based generation scheme, the distributed generation (DG) inverters will be remotely connected [8]. In such a case, it is impractical to distribute a high bandwidth signal for dynamic sharing. On the other hand, parallel operation of low-power converters in the same equipment cabinet can be accomplished through distribution of high bandwidth control signals provided that the internal electromagnetic-compatibility issues are addressed. The "power park" arrangement of distributed generators lies somewhere between these two extremes. The power rating of the units means they will not be in the same equipment cabinets but they will be only a few meters apart and their inverters will export power through a common generator bus. In such a case, the sharing of control signals will require a communication bus such as controller area network (CAN) Bus, FireWire, or 


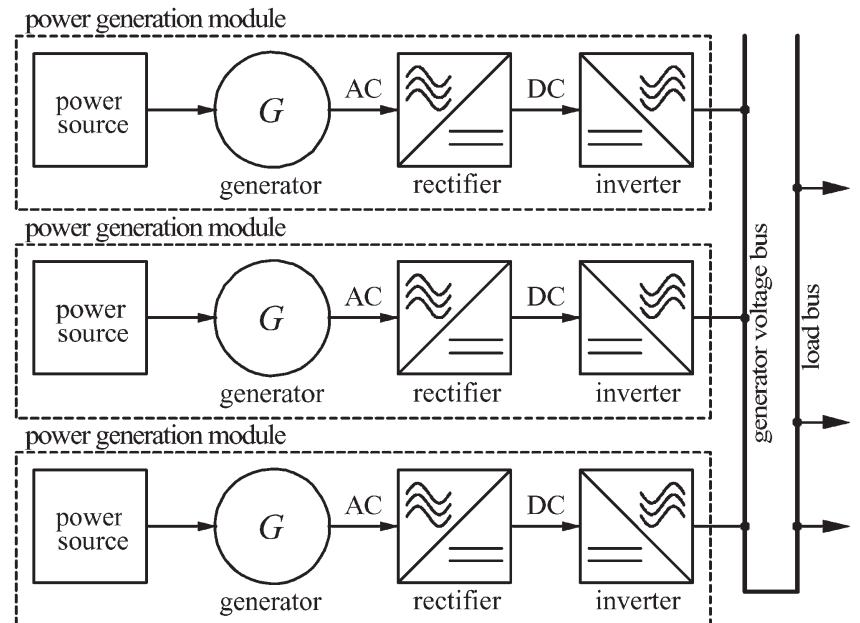

Fig. 1. Modular approach in power generation.

ProfiBus. With such a communication link in place, control methods can be adopted that seek to provide dynamic sharing between the inverters [2], [3], [5], [9]. In the case of a power park and a explicit communication link, it is important to consider the bandwidth over which dynamic sharing is being sought and the limitations imposed by the implementation of the communication link.

The control objectives for DG inverters can be classified by priority and by frequency range. The prime objective is the export of real power and to have this duty shared equally between the units. This is a steady-state regulation issue. Where local voltage control is allowed, regulation of the voltage magnitude is also required. This will be the case in a microgrid running in island mode or running gird connected via a large valued coupling impedance. The voltage control may be extended to include control of voltage balance between the three phases, which can be expressed as suppression of zero- and negativesequence components of voltage caused by unbalanced current flows. Consideration of power quality gives a further set of control objectives. Power quality is a particular concern in small-scale islanded systems because nonlinear and singlephase (unbalanced) loads can represent a high proportion of the total load and in a relatively weak system can have a strong impact on the voltage waveforms. Further, switching of a single load can represent a large transient that can cause sag and swell problems. The power quality expected of distributed generators has been the subject of discussion and standards have emerged [10]. The general principles are discussed in [11] where it is stated that in a voltage source system, the power quality is defined by the voltage quality. Standards for harmonic distortion of the loads connected to a voltage grid are covered in [12].

This paper will examine the control, including power-quality control, of a system of inverters connected in parallel in close electrical proximity. This means that the generators are connected to a common bus before connection to the distribution network or microgrid (Fig. 1). The case considered is one in which the DG system has control over the local voltage such as occurs in island operation. The proposed control topology uses a communication link of limited bandwidth to maintain power sharing between units. The control objective is high power qual- ity under linear, nonlinear, balanced, unbalanced, and transient loads. The principle adopted is to distribute the control between a central controller and local controllers for each inverter. The control action is partitioned in the frequency domain. Steady-state and low-frequency (principally unbalance) issues are controlled centrally and a low-bandwidth communication channel is employed to distribute control signals to individual units. High-frequency issues, such as harmonic suppression, are dealt with locally to each inverter without the use of a communication channel. Thus, communication is used where it can have most effect and where the bandwidth requirement will not be onerous or expensive to implement. The intention is to achieve approximately the same control performance from a set of parallel units as could be obtained from a single unit of the combined rating. The theoretical basis for the frequency partition will be discussed and its performance analyzed. The proposal is then tested through an experimental implementation using three 10-kVA inverters and a set of example loads.

\section{PARAllel Connection of Inverters}

In the example system of Fig. 1, the power sources considered might be wind turbines or high-speed gas turbine generators that generate ac voltage with a frequency different from 50 or $60 \mathrm{~Hz}$. The ac voltage will be rectified to form a dc bus and an inverter used to export power to the generator ac bus for onward distribution of power to the rest of the network. The specifications and restrictions on the design of the control system will now be examined.

\section{A. Steady-State Regulation}

The control of the parallel connected units must satisfy two main objectives: voltage regulation and power sharing between the modules. The voltage regulation role is to maintain the three-phase voltages and frequency at a certain level under various balanced and unbalanced, linear, and nonlinear loads. The power-sharing feature has to provide that all modules share the load according to their ratings and availability. Since there is one common ac bus voltage (the generator bus), the control of voltage magnitude and frequency is common to all units and it is natural to choose to control this centrally. Power sharing must also be a central task since some measure of the aggregate load must be made and then shared.

\section{B. Waveform Quality Control}

The fast response of an inverter facilitates the rejection of disturbances such as harmonic currents caused by nonlinear loads and negative- and zero-sequence currents caused by single-phase loads. It is expected that an inverter should give much better disturbance rejection than a synchronous machine in which there is no active control of these factors. This is relatively easy to achieve with a single inverter system but the reported methods of paralleling inverters do not fully exploit the capabilities inverters in this respect [6], [13]. In a microgrid with a high proportion of nonlinear loads, the supply of harmonic current to the loads should be shared between the units. This will avoid one inverter being subject to excessive rms 


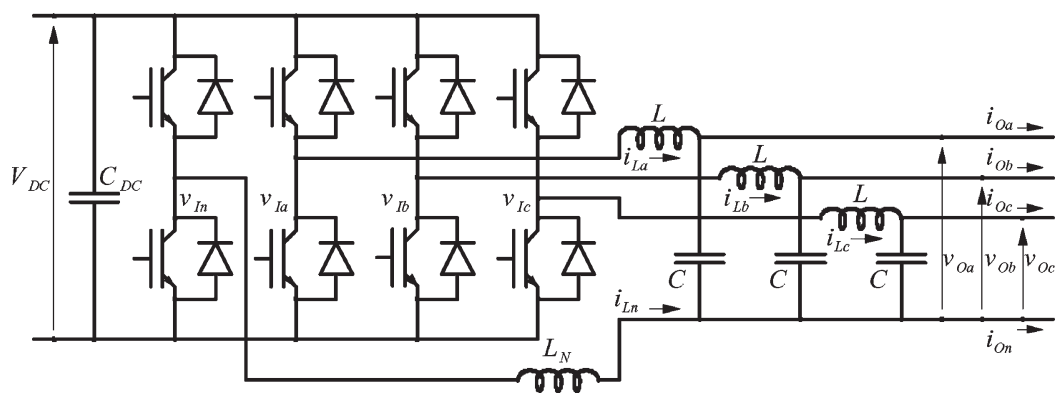

Fig. 2. Four-leg voltage-source inverter.

current but small real power. The sharing of harmonic current requires that the inverters share the action of suppressing the harmonic voltage at the common bus. Because the suppression of harmonics will require a high-bandwidth feedback and this is best handled locally by each inverter. The sharing of harmonic duty is then on the basis of the units being reasonably matched and having equivalent control characteristics.

Control of voltage balance in the presence of a large number of single-phase loads is also necessary. Unbalance is usually expressed in terms of the magnitude of the negative- and zerosequence components [14]. Existing control methods exhibit various responses to unbalanced load [5], [6]. Ideally, the system should share the unbalance and operate as if it were a combination of three independent single-phase systems in which the phase voltage magnitudes and the angles between the voltages are individually maintained. Suppression of negativeand zero-sequence disturbances is more than a steady-state regulation problem and requires reasonable bandwidth. It could be allocated as a central or a local control task depending on the bandwidth that can be realistically implemented by the interunit communication link.

\section{Transients}

The switching of loads in a small scale power system can cause relatively large transient errors in the voltage since the switched load may represent a large fraction of the total load. The sag or swell of voltage, and the recovery time following a load change should be small and the transient oscillations must be well damped and free of circulating currents [7], [15].

In control methods based on the master-slave concept [2], [3], [5], [16], only one unit controls the voltage and provides (or absorbs) all of the transient power in the first instance. In other approaches [2], [3], [17], the transient current is shared equally. In a distributed control system, both the local and central control loops will act during the transient. The highbandwidth local controllers will dominate the initial response and the central controller will dominate the closing stages of transient response.

\section{Communication Links}

The existing methods for control of a parallel-inverter system can be divided in those that use a communication link, and those that do not, to achieve voltage control and power sharing. For inverters that are remotely connected, methods that do not use communication have an obvious advantage in terms of

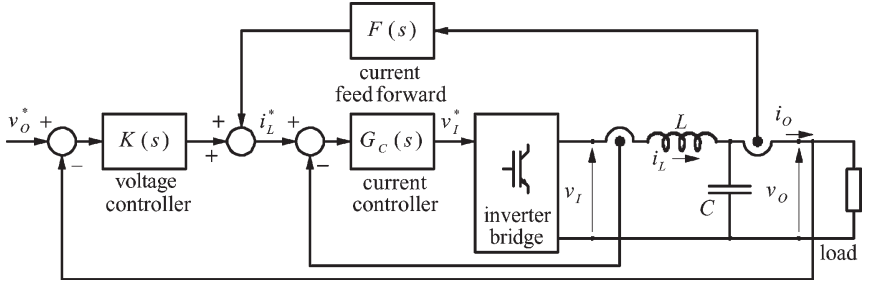

Fig. 3. Per-phase structure of conventional voltage control loop.

cost and reliability. The methods [6], [7], [13] are based on mimicking the operation of the synchronous generators. The disadvantage of these methods, especially in small-scale systems, is the inability to provide rejection of voltage harmonic content and control zero-sequence unbalance. When inverters are closely connected, the introduction of a communication link can improve the performance of the system. In [17], it was shown that the power-sharing and power-quality performance depend on the bandwidth of the communication link.

\section{Conventional Control of a Single Inverter}

Since the objective of distributed control is to obtain from a group of inverters the same performance as a single inverter, it is worth reviewing how a single inverter would be controlled. A four-wire network will be used if the loads include single-phase loads and the inverter will need to be a four-leg design if full control of neutral current and full utilization the dc-link voltage are desired [14]. As Fig. 2 shows, the inverter is followed by a low pass filter to remove voltage components generated by the inverter switching and to reduce the ripple of the inverter output currents. For a four-leg inverter, a neutral inductor can be added to increase the inductance presented to common mode (zero-sequence) components.

Closed-loop control of the voltage at the output of the filter is commonly achieved with the two-loop structure shown in Fig. 3 and discussed in [18] and [19]. An inner current control loop is placed around the inductor and voltage source inverter to form a controlled current source. An outer voltage control loop acts on the voltage error to set a current demand for the inner loop.

The objective of the voltage control loop is to provide good tracking of slow changes in the reference signal $v_{O}^{*}$ and to minimize errors in the output voltage caused by disturbances introduced by changes in the load current $i_{O}$. Disturbance rejection is provided by both the feedback of the voltage $v_{O}$ and the feedforward of the load current $i_{O}$. For an inverter under closed-loop control, the output impedance is defined by (1), 


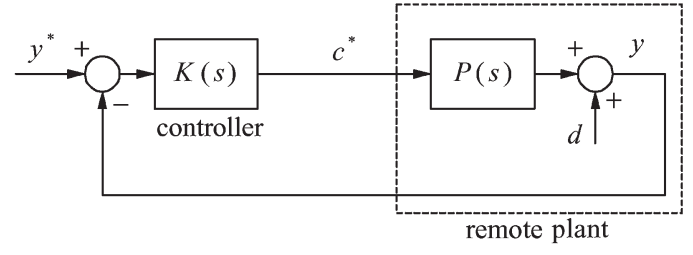

Fig. 4. Conventional control method.

where $G_{\mathrm{S}}(s)$ is the closed-loop transfer function of the inner current control loop.

$$
Z_{O}(s)=\frac{V_{O}(s)}{I_{\mathrm{O}}(s)}=\frac{1}{s C} \cdot \frac{\left(F(s) G_{\mathrm{S}}(s)-1\right)}{\left(1+K(s) G_{\mathrm{S}}(s) \frac{1}{s C}\right)}
$$

A low impedance at a particular frequency represents good rejection of disturbances occurring at that frequency. The impedance can be decreased either by increasing the loop gain (the second denominator term) or by minimizing the difference term in the numerator. The gain of the current control loop $G_{\mathrm{S}}(s)$ will be close to unity across the bandwidth of interest if it has been properly designed. The gain of the feedforward transfer function $F(s)$ is also unity or a little less within the control bandwidth.

The feedforward and feedback terms combine to set the current reference for the inner loop and is given by (2). The control signal $i_{L}^{*}$ will use all available bandwidth to minimize the effect of the disturbance on the output voltage. This has important implications for control of a parallel connection of inverters: If the communication bandwidth is limited, the disturbance rejection will be compromised

$$
\frac{I_{\mathrm{L}}^{*}(s)}{I_{O}(s)}=\frac{F(s)+K(s) \frac{1}{s C}}{1+K(s) G_{\mathrm{S}}(s) \frac{1}{s C}} .
$$

In a standard control loop, such as Fig. 4, the controller $K(s)$ is connected directly to the plant $P(s)$ and the drive signal $c^{*}$ is not subject to any bandwidth constraint. In the case of DG inverters, the plant is an inverter and its local current controller. The controller we need to discuss is the voltage controller.

In the case where the plant (inverter) is connected remotely from the (voltage) controller, the bandwidth with which the drive signal is distributed is of importance and will determine the limit of the high-frequency components of the output signal being controlled.

Equations (3) and (4) express the transfer functions from an output disturbance to the output itself and the drive signal. This shows that if the controller is designed to give good and rapid disturbance rejection, the drive signal will need to have a wide bandwidth

$$
\begin{aligned}
R(s) & =\frac{Y(s)}{D(s)}=\frac{1}{1+K(s) P(s)} \\
\frac{C^{*}(s)}{D(s)} & =-\frac{K(s)}{1+K(s) P(s)} .
\end{aligned}
$$

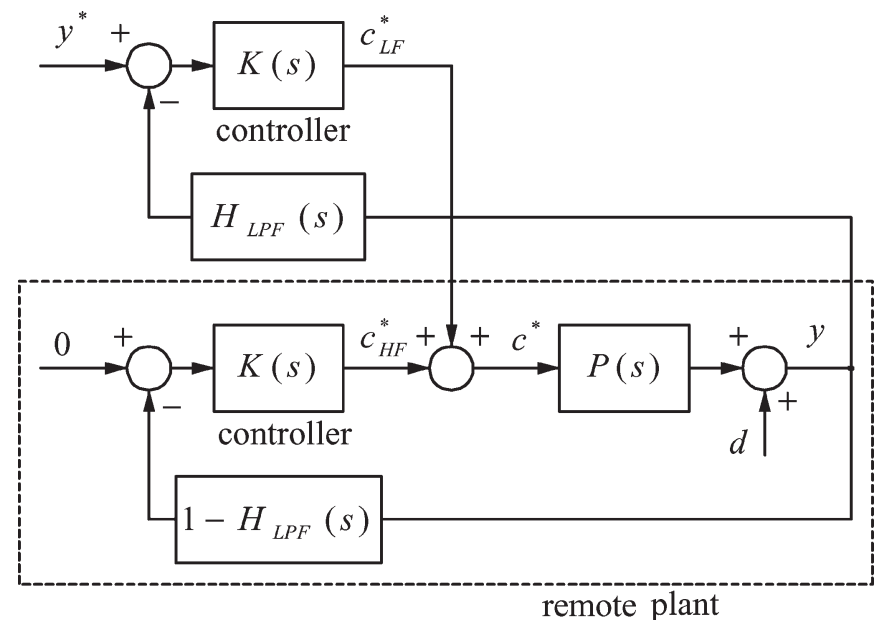

Fig. 5. Distributed control method.

\section{Distributed Control Through FREQUENCY PARTITION}

The principle of distributed control is to divide the control action between the central controller and the local controllers through a frequency partition: the central controller is responsible for ensuring proper following of a reference voltage that is by its nature limited to low-frequency terms and the local controllers are responsible for rejecting high-frequency disturbances, [17], [20]. The signal distributed from the central controller to local units is only the low-frequency part of the drive signal and can be passed through a communication link of limited bandwidth. This new control structure is shown in Fig. 5 for the simple case of a central controller and one unit. By introducing the low-pass filter $H_{\mathrm{LPF}}$ and matched high-pass filter $\left(1-H_{\mathrm{LPF}}\right)$ to the feedback signal, the control spectrum is perfectly shared between two controllers.

The drive signal $c^{*}$ is formed by adding low-frequency $c_{\mathrm{LF}}^{*}$ and high-frequency $c_{\mathrm{HF}}^{*}$ components. The reference signal for the higher frequency components is zero since only disturbance rejection is required in this frequency range. Provided that the filters are perfectly matched and that the central and local controllers together are properly chosen, the performance of the distributed control system can match that of a single controller with unlimited communication bandwidth. In particular, the disturbance transfer function $R(s)$ of the new system will be the same as that of the conventional control structure previously shown in (3) if the local and remote controllers have the same transfer function.

The bandwidth requirement for the link between the central controller and the individual units can be can be found by analyzing the partition of the drive signal $c^{*}$. The choice of the low-pass filter $H_{\mathrm{LPF}}(s)$ is a design tradeoff. In order to use a standard industrial communication channel it is desirable to have a low cutoff frequency for the partition filter so as to limit the bandwidth required. However, by maintaining a high bandwidth in the central control loop, more of the control effort in a transient response is subject to explicit sharing and less to local action subject to unit mismatch. The low- and highfrequency drive signals are given in (5) and (6). The product of $K(s) H_{\mathrm{LPF}}(s)$ shapes the frequency content of the central drive 


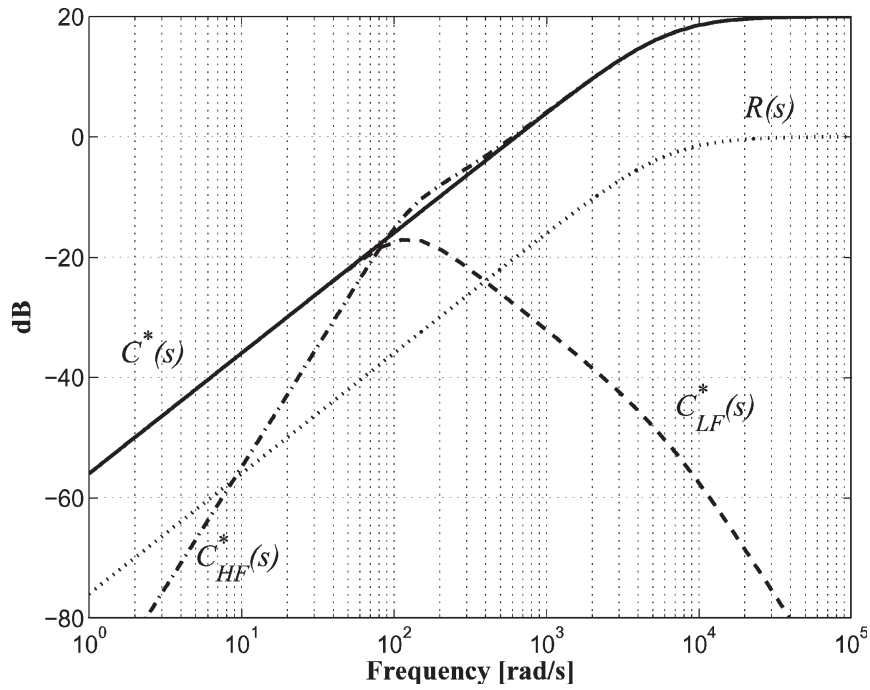

Fig. 6. Control signal bandwidth and disturbance rejection transfer functions.

signal. It is important to ensure that the complimentary product, $K(s)\left(1-H_{\mathrm{LPF}}(s)\right)$ has a low gain in the low-frequency range to prevent the local plant controller taking part in steady-state and low-frequency regulation

$$
\begin{aligned}
\frac{C_{\mathrm{LF}}^{*}(s)}{D(s)} & =-\frac{K(s) H_{\mathrm{LPF}}(s)}{1+K(s) P(s)} \\
\frac{C_{\mathrm{HF}}^{*}(s)}{D(s)} & =-\frac{K(s)\left(1-H_{\mathrm{LPF}}(s)\right)}{1+K(s) P(s)} .
\end{aligned}
$$

An example will be used to demonstrate the advantage of the distributed control method. A proportional controller was designed to control a plant, which included integral action (a plant similar to the capacitor of an inverter output filter). The transfer functions of the controller and the plant are given in (7). The partition filter, $H(s)$ was a second-order low-pass Butterworth filter with a cutoff frequency of $20 \mathrm{~Hz}$

$$
K(s)=10 \quad P(s)=\frac{2 \pi 100}{s}
$$

Fig. 6 shows the transfer functions for several signals with respect to an output disturbance $D(s)$. The dotted line shows the disturbance rejection ratio $R(s)$ (the transfer function from disturbance to output). This rejection achieved with the drive signal (control effort) $C^{*}(s)$ shown by the solid line which is composed of low-frequency $C_{\mathrm{LF}}^{*}(s)$ and high-frequency $C_{\mathrm{HF}}^{*}(s)$ portions. The control effort required rises with frequency because the plant itself had a low pass (integral) nature which reduces the loop gain with frequency. For this reason, it is important that the control effort is not bandwidth limited. What the figure makes clear is that the frequency partition allows $C_{\mathrm{LF}}^{*}(s)$ to be of limited bandwidth (such that it could be transmitted over a standard industrial communication link) without limiting the bandwidth of the complete drive signal and without degrading the disturbance rejection properties of the whole system.

\section{Distributed Control Applied to a Power Park OF DG INVERTERS}

The objective is to apply the controller structure of Fig. 3 to the example power park of Fig. 1. The inverters represent a set of local plant. Each has its own internal current control loop and so the inverters can be considered as controlled current sources. The voltage control loop requires a low-frequency regulation function that is common to all and achieves sharing of the control effort between units. Fig. 7 shows the arrangement of control loops for the case of a central controller and three inverters. The central controller takes voltage feedback $v_{O}$ from the generator bus common to all inverters of the power park. The current $i_{O}$ drawn from the generator bus by the loads is used as the feedforward term. The control effort is the current demand signal $i_{L}^{*}$ and the low-frequency portion of this, $i_{L \mathrm{LF}}^{*}$, will be generated centrally and shared between units across a limited bandwidth communication link. Sharing of this signal ensures that fundamental frequency real and reactive power are shared between the units regardless of mismatches in inverter properties. The control effort of supplying unbalance current and providing transient response can also be explicitly shared if the bandwidth of the central controller and communication link permit this otherwise they will be subject to independent local control.

The inverters contain a local controller that is principally responsible for power-quality improvement. It acts on local feedback of the bus voltage (and a zero reference). Its highfrequency response is used to suppress harmonic and transient disturbances to the bus voltage caused by the load current. The partition of the control bandwidth between the central and local controllers maintains disturbance rejection properties across a wide range of frequency, limited only by the switching frequency of the inverter.

If the inverters are distributed and there is no means of sensing the total output current of the system (unlikely in a power park case), the same structure should used and the feedforward terms should be applied only locally within the inverter controllers, but not in the central controller. This will compromise the dynamic response under transient load change, but will not affect neither the voltage regulation nor steady-state power sharing (that are controlled by the central controller).

\section{A. Connection of Inverters With Different Ratings}

A common situation would be a power park in which there is a mix of generation types or sizes. The sharing of the real, reactive, and unbalance powers needs to be performed with regard to the ratings of the units. Distributed control can be applied by introducing a scaling factor into the distribution of the low-frequency current reference. The scaling factor can be defined as the ratio of current ratings. As an example, if two inverters of 20 and $10 \mathrm{kVA}$ are to be connected in parallel, their nominal currents will be in ratio $1: 2$. The low- and highfrequency inductor current references of the 20-kVA unit need to be multiplied by 2 and those for the other inverter by 1 (taking $10 \mathrm{kVA}$ to be the base unit). The feedforward scaling in the central controller will be set to $2 / 3$ and $1 / 3$ for the two units. A further stage of matching would be to design the inverters for 


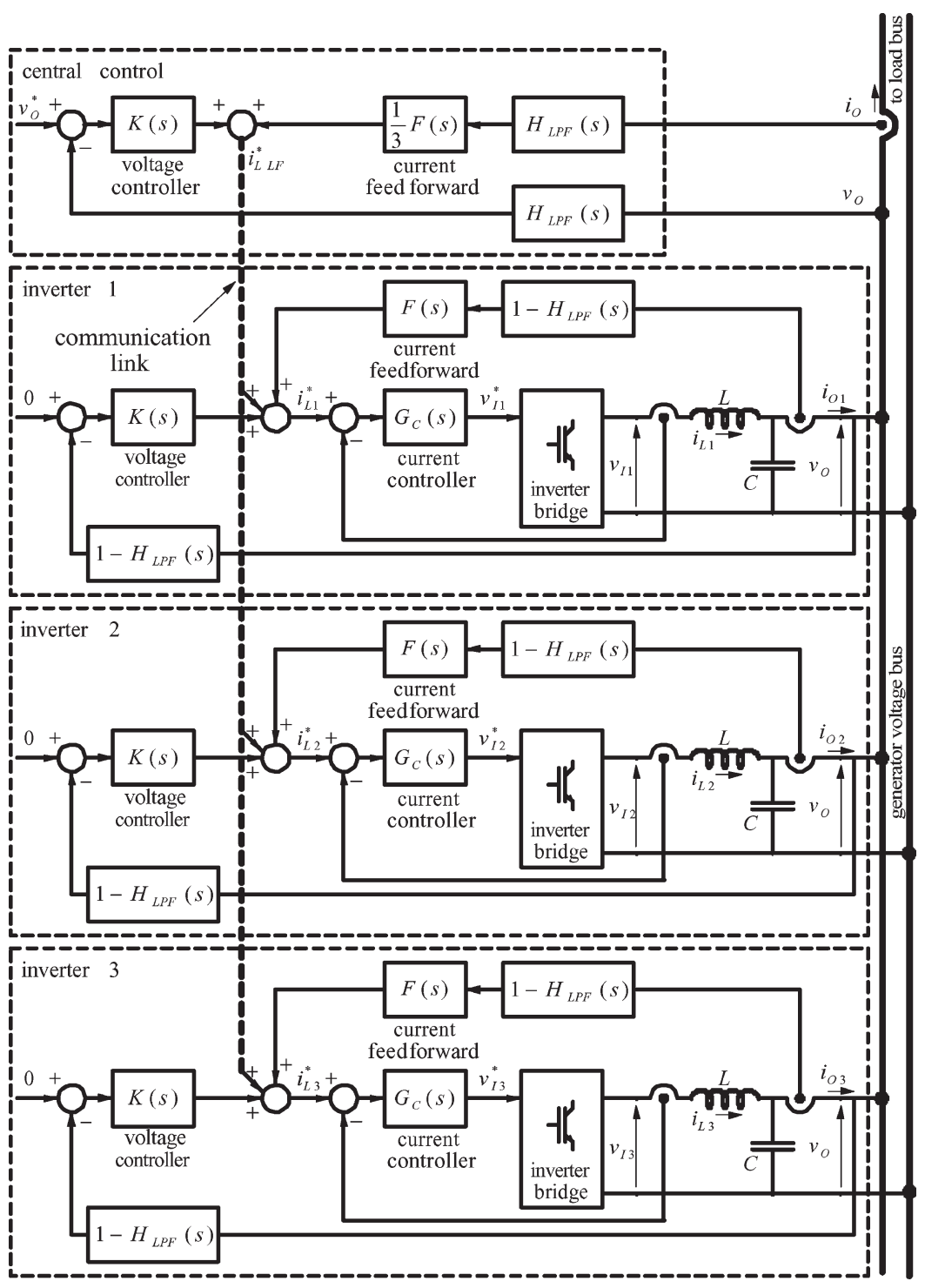

Fig. 7. Application of discontinuous conduction mode to parallel inverter system.

the same switching frequency and the same voltage ripple and current ripple. In this case, the filter capacitances would be in the ratio $2: 1$ and the dynamic properties of the two units will be equivalent and the systems over all dynamics will match that of the equivalent rating of single inverter.

For a system of $N$ identical parallel-connected inverters, the overall output impedance is given by (8). The same formula can be applied to an inverter system of nonidentical units if their current control loop transfer functions $G_{\mathrm{S}}(s)$ are the same and their output capacitances scaled. The capacitance $C$ would then represent the capacitance of the base unit that was used for the control design and $N$ would be defined as the ratio between the total output capacitance and the capacitance of the base unit. The output impedance in (8) is $N$ times smaller than the impedance of the single unit defined in (1). This indicates better rejection of disturbances results from the increased equivalent capacitance in the system

$$
Z_{O}(s)=\frac{V_{O}(s)}{I_{O}(s)}=\frac{1}{N} \frac{1}{s C} \frac{\left(F(s) G_{\mathrm{S}}(s)-1\right)}{1+K(s) G_{\mathrm{S}}(s) \frac{1}{s C}} .
$$

The bandwidth required for the shared reference signal, $i_{L L F}^{*}$, can be estimated for the multiple inverter case from (9). The corresponding bandwidth for the local signal is given in (10) for completeness

$$
\begin{aligned}
\frac{I_{\mathrm{LLF}}^{*}(s)}{I_{O}(s)} & =\frac{1}{N} \frac{F(s)+K(s) \frac{1}{s C}}{1+K(s) G_{\mathrm{S}}(s) \frac{1}{s C}} H_{\mathrm{LPF}}(s) \\
\frac{I_{\mathrm{LHF}}^{*}(s)}{I_{O}(s)} & =\frac{1}{N} \frac{F(s)+K(s) \frac{1}{s C}}{1+K(s) G_{\mathrm{S}}(s) \frac{1}{s C}}\left(1-H_{\mathrm{LPF}}(s)\right) .
\end{aligned}
$$

By comparing (9) and (10) to the equation for a single unit given in (2), it can be seen that the distributed control system is equivalent except for a factor of $1 / N$ because the plant transfer function has a factor of $N$ that arises from the multiple current sources operating in parallel.

\section{B. Robustness Analysis}

It is important to examine the robustness of the control strategy to variation of the parameters of the various inverters 
and to measurement error which may lead to unequal sharing of power between units and the development of circulating currents. For the worst case scenario, it is assumed that the first unit in Fig. 7 has a disturbance applied to its output in the form of an output voltage measurement error of $\Delta v_{O}$. The responses of the output voltage $v_{O}$ and inductor currents $i_{L 1}, i_{L 2}$, and $i_{L 3}$ are shown in (11)-(13)

$$
\begin{aligned}
& \frac{V_{O}(s)}{\Delta V_{O}(s)}=-\frac{1}{N} \frac{G_{\mathrm{S}}(s) K(s)\left(1-H_{\mathrm{LPF}}(s)\right) \frac{1}{s C}}{1+K(s) G_{\mathrm{S}}(s) \frac{1}{s C}} \\
& \frac{I_{L 1}(s)}{\Delta V_{O}(s)}=-\frac{1}{N}\left(\frac{(N-1) G_{\mathrm{S}}(s) K(s)\left(1-H_{\mathrm{LPF}}(s)\right)}{1-G_{\mathrm{S}}(s) F(s)\left(1-H_{\mathrm{LPF}}(s)\right)}\right. \\
& \left.+\frac{G_{\mathrm{S}}(s) K(s)\left(1-H_{\mathrm{LPF}}(s)\right)}{1+K(s) G_{\mathrm{S}}(s) \frac{1}{s C}}\right) \\
& \frac{I_{L 2,3}(s)}{\Delta V_{O}(s)}=-\frac{1}{N}\left(-\frac{G_{\mathrm{S}}(s) K(s)\left(1-H_{\mathrm{LPF}}(s)\right)}{1-G_{\mathrm{S}}(s) F(s)\left(1-H_{\mathrm{LPF}}(s)\right)}\right. \\
& \left.+\frac{G_{\mathrm{S}}(s) K(s)\left(1-H_{\mathrm{LPF}}(s)\right)}{1+K(s) G_{\mathrm{S}}(s) \frac{1}{s C}}\right) .
\end{aligned}
$$

The condition that must be satisfied for the system to be stable and to prevent circulating currents is that the feedforward gain should less than the term given in (14). With careful choice of the controller and filter transfer functions, steady-state power sharing can be assured

$$
F(s)_{\max }<\left|\frac{1}{G_{\mathrm{S}}(s)\left(1-H_{\mathrm{LPF}}(s)\right)}\right|_{\max } .
$$

\section{Distributed Control in a Rotating Reference Frame}

The rotational reference frame (variables $d, q$, and 0 ) has advantages over a stationary reference frame (variables $d, q$, and 0) for control of a three-phase multi-inverter system [21]. In a balanced and undistorted system, components of the voltage vector appear as dc variables and standard controller designs can be readily applied to their regulation. Instead of tracking three fundamental frequency references, only three dc levels need to be regulated. In the context of distributed control, this means that the shared control signal need not be a fundamental frequency signal but instead a set of dc quantities representing fundamental components. This leads to further reduction of the communication bandwidth requirement.

If negative-sequence unbalance is present in the load, the $d$ and $q$ components of the output current have a twice fundamental frequency component (representing the unbalance) in addition to the de terms (representing total system active and reactive power demand). Zero-sequence unbalance causes fundamental frequency content in the zero-voltage component. In order to imbalance disturbance to the output voltage, these additional components must be rejected by high loop gain. This can be chosen to be either a central or a local function according to the communication bandwidth available for use.

To implement distributed control in a rotational reference frame, it is necessary to use the same reference frame throughout the system. This requires a periodic synchronization signal to be distributed together with the low-frequency portion of the drive signal.
The dominant dynamics of the power park system are those of the inverter filters. The state-space model of the four-wire filter in Fig. 2 was derived. The sixth-order state-space model, expressed as derivatives of the state variables $i_{\mathrm{L}}$ and $v_{O}$, is given by (15) and (16). The system inputs are inverter bridge voltages $v_{I}$ and load currents $i_{O}$. The presence of the fourthleg of the filter makes control in a stationary reference frame complicated because of the various coupling terms. In the rotational transformation the coupling terms can be readily dealt with by adding decoupling control. Details of the discretization and decoupling of this system are provided in [18]

$$
\begin{aligned}
{\left[\begin{array}{c}
i_{L d} \\
i_{L q} \\
i_{L 0}
\end{array}\right]=} & {\left[\begin{array}{ccc}
0 & \omega L & 0 \\
-\omega L & 0 & 0 \\
0 & 0 & 0
\end{array}\right]\left[\begin{array}{l}
i_{L d} \\
i_{L q} \\
i_{L 0}
\end{array}\right] } \\
& +\left[\begin{array}{ccc}
\frac{1}{L} & 0 & 0 \\
0 & \frac{1}{L} & 0 \\
0 & 0 & \frac{1}{L+3 L_{n}}
\end{array}\right]\left[\begin{array}{l}
v_{I d}-v_{O d} \\
v_{I q}-v_{O q} \\
v_{I 0}-v_{O 0}
\end{array}\right] \\
{\left[\begin{array}{c}
v_{O d} \\
v_{O q} \\
v_{O 0}
\end{array}\right]=} & {\left[\begin{array}{ccc}
0 & \omega C & 0 \\
-\omega C & 0 & 0 \\
0 & 0 & 0
\end{array}\right]\left[\begin{array}{l}
v_{O d} \\
v_{O q} \\
v_{O 0}
\end{array}\right] } \\
& +\left[\begin{array}{ccc}
-\frac{1}{C} & 0 & 0 \\
0 & -\frac{1}{C} & 0 \\
0 & 0 & -\frac{1}{C}
\end{array}\right]\left[\begin{array}{l}
i_{O d}-i_{L d} \\
i_{O q}-i_{L q} \\
i_{O 0}-i_{L 0}
\end{array}\right] .
\end{aligned}
$$

The proposed control structure shown in Fig. 7 is applied to all axes through three parallel control loops acting on the $d, q$, and 0 components of the output voltage.

\section{Example of Distributed Control of a Multiple Inverter System}

There are several issues to consider in deciding how to partition the bandwidths of the central and local control loops, and some design tradeoff is necessary.

1) The bandwidth of the internal current controllers should be set as high as possible to create near ideal controlled current sources. In practice, this will be limited by the switching frequency and the controller-sampling period.

2) The bandwidth of the composite voltage controller should be as high as possible to provide rejection of harmonic distortion.

3) The loop gain at dc should be as high as possible to control the magnitude of the fundamental frequency voltage with low error.

4) The loop gains at fundamental and twice fundamental frequency should be high in order to suppress imbalance.

Design tradeoffs have to be made in shaping the frequency response of $K(s)$. For example, increasing the gain helps reduce output impedance at critical frequencies and increases voltage control bandwidth, but reduces phase margin in the naturally resonant $L C$ system.

The current feedforward transfer function $F(s)$ helps reduce the voltage error under a disturbance and also reduces the output impedance. It is normal to include a low-pass filter response in $F(s)$ because the feedforward term can introduce high-frequency components into the system. The cutoff 
frequency of this filter should be well within the current control bandwidth.

It is recommended that the controller partition filter $H_{\mathrm{LPF}}(s)$ be designed with a cutoff frequency in the range of $5-20 \mathrm{~Hz}$. This attenuates the double fundamental frequency component in the central control so as to leave rejection of unbalance disturbance to the local inverter controllers. This choice of partition frequency is still high enough to ensure a response time of few cycles to any transient disturbance (load switching) such that mismatches of transient response between the inverters is quickly corrected and circulating currents avoided.

An example system of three inverters connected in parallel will be used to demonstrate the properties of distributed control. The controller design procedure for the whole system is the same as it would be for a single inverter optimized for the maximum dynamic performance. The detailed background theory for the discrete-time controller design for power inverters has been discussed in [18], [22]-[24]. From the control structure shown in Fig. 3, the open loop transfer function of the voltage control is derived as in (17). The transfer function assumes no-load condition (worst case scenario for the stability) and consists of the series connection of the voltage controller, current controller $G_{\mathrm{S}}(s)$ and inverter filter capacitance of $C=50 \mu \mathrm{F}$

$$
W(s)=K(s) G_{\mathrm{S}}(s) \frac{1}{s C} .
$$

By using a realistic assumption for the switching frequency of $8 \mathrm{kHz}$ and applying the estimators to improve the control bandwidth as in [23] and [24], the closed-loop response of the internal current control can be approximated in the continuous time domain as in (18)

$$
G_{\mathrm{S}}(s)=\frac{1}{s /(2 \pi 2000)+1} .
$$

To obtain the best dynamic performance of the voltage regulation (high-voltage control bandwidth and low output impedance), the maximum possible proportional and integral gains of the voltage controller $K(s)$ should be selected that provide the stable response. The feedforward gain must satisfy the condition (14). The selected transfer functions for the voltage controller and feedforward terms are given in (19)

$$
K(s)=0.2+\frac{300}{s} \quad F(s)=0.3
$$

By assuming that the digital communication link between the central controller and inverters delivers the low-frequency current reference once every $2 \mathrm{~ms}(500 \mathrm{~Hz})$, a second-order low-pass Butterworth filter with a cutoff frequency of $20 \mathrm{~Hz}$ is chosen to provide sufficient attenuation of the frequency content of the reference signal (higher than $250 \mathrm{~Hz}$ ) before the decimation. Because the product $K(s)\left(1-H_{\mathrm{LPF}}\right)$ would have nonzero dc gain, an additional first-order high-pass filter of $10-\mathrm{Hz}$ cutoff frequency is added in series to the filter $(1-$ $\left.H_{\mathrm{LPF}}\right)$ to prevent the local inverter controllers participating in control of the fundamental frequency voltage component. This does not affect the design principle of the filter and controllers.

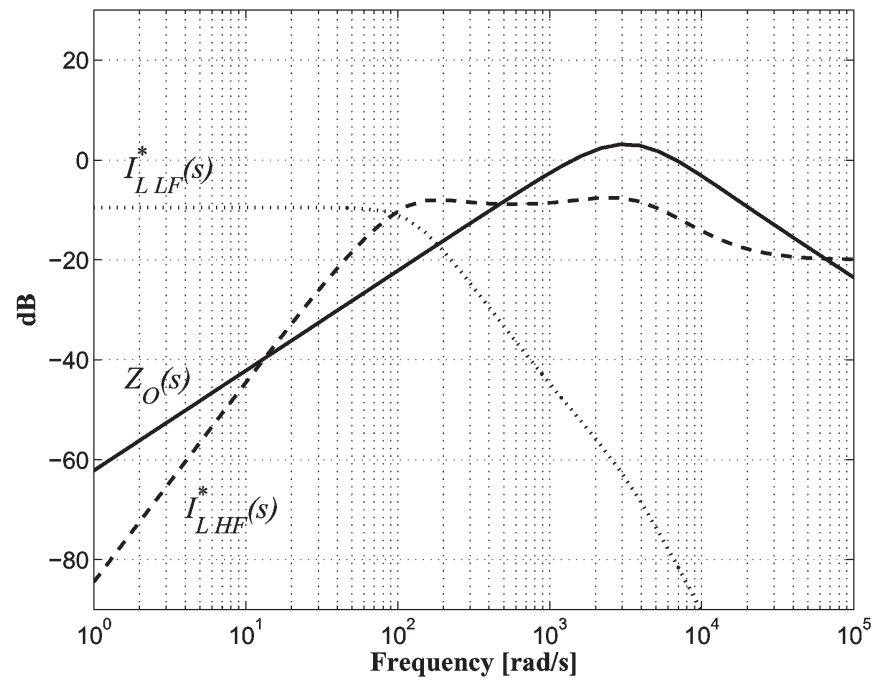

Fig. 8. Low- and high-frequency reference and output impedance $Z_{O}(s)$ transfer functions.

Fig. 8 shows the overall output impedance of the system $Z_{O}(s)$ together with the transfer functions of low- and highfrequency portions of the control drive signals $I_{\mathrm{LLF}}^{*}(s)$ and $I_{\mathrm{LHF}}^{*}(s)$ with respect to output current disturbance $I_{O}(s)$. The low-frequency drive signal has a limited bandwidth that is convenient for sharing between the units. The output impedance indicates good rejection of output current disturbance. The low value of $Z_{O}(s)$ at low-frequency indicates that that the steadystate voltage will not be affected by the load present.

The robustness of the overall control to parameter variation was assessed through measurement error $\left(\Delta v_{O}\right)$ applied to one of the units. This represents the worst case parameter variation, since the variation of the inverter filter parameters and/or voltage controller gains is compensated either locally by the current control loop or by the overall voltage control loop. Since the steady-state voltage is controlled from the central controller, any variation of these parameters does not affect neither the steady-state voltage control nor the power sharing.

Bode plots of the response of the output voltage $V_{O}(s)$, the current of the unit in error $I_{L 1}$, and current of the other units $I_{L 2}=I_{L 3}$ are shown in Fig. 9. The corresponding transfer functions are shown in (11)-(13). The magnitude of the response to a low frequency or dc error is very low and the steadystate power sharing will not be adversely affected. In this way, circulating currents are avoided even when measurement error is present in the local controller. However, some noticeable difference in current sharing at higher frequencies may exist. The sharing of harmonic current then depends on the degree of measurement error (or parameter variation) and on the control design parameters. It can be seen that the output voltage is less affected than the inductor currents.

\section{EXPERIMENTAL RESULTS}

An experimental rig was built with three 10-kVA threephase inverters connected in parallel. The inverter switching frequency and filter parameters where chosen to be representative of real microturbine DG systems in the 100-kVA 


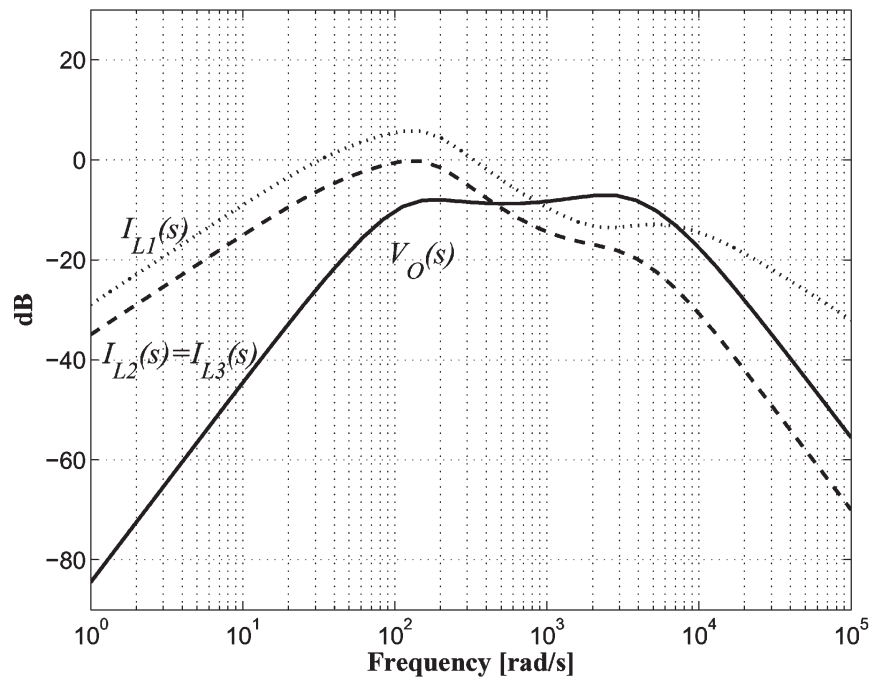

Fig. 9. Bode plots of impulse response of $V_{O}, I_{L 1}, I_{L 2}$, and $I_{L 3}$ to $\Delta V_{O}$.

range so that realistic limits were placed on the control system performance. The inverter parameters were:

1) nominal rms phase voltage of $220 \mathrm{~V}$;

2) switching frequency $f_{\mathrm{S}}=8.192 \mathrm{kHz}$;

3) inverter filter parameters, $C=50 \mu \mathrm{F}, L=1.35 \mathrm{mH}$, and $L_{n}=0.45 \mathrm{mH}$

4) current control loop bandwidth of $1.9 \mathrm{kHz}$;

5) voltage control loop bandwidth of $500 \mathrm{~Hz}$;

6) feedforward bandwidth of $1.5 \mathrm{kHz}$ with gain of 0.3 ;

7) control partition filter, $H_{\mathrm{LPF}}$ of $20 \mathrm{~Hz}$ cutoff, and secondorder Butterworth characteristic;

8) communication link CAN bus with reference update time of $2 \mathrm{~ms}$.

The controllers were implemented on TMS320LF2407 processors in both the central controller and the inverter units. These processors also generated the pulsewidth-modulation signals to drive the inverter.

Transient power sharing is of particular importance in a set of parallel connected inverters. A measure of the transient power quality can be expressed as the time required for the system to recover from the transient and the trajectory of the controlled variable during its progress to steady state. Fig. 10(a) and (b) show the output voltages and currents during a $0 \%$ to-100\% step of a balanced resistive load. The transient response lasted for only $2 \mathrm{~ms}$. The control showed good stability with no oscillatory behavior. After the transient, the system entered steady state and showed the characteristics of a wellbalanced three-phase system. The phase voltage and current levels were $220 \mathrm{~V}_{\mathrm{rms}}$ and $42 \mathrm{~A}_{\mathrm{rms}}$, respectively. The total output power was $28 \mathrm{~kW}$. There was negligible difference between the magnitudes of the three-phase voltage and the phase shifts between them were $-119.8^{\circ}$ and $120.1^{\circ}$.

The transient power sharing is demonstrated in Fig. 10(c) which shows the phase- $A$ inductor current of all three inverters. Before the transient, the inductor currents consisted of only the switching frequency components and a small fundamental frequency component flowing in the filter capacitor. After the transient, the load current was dominant. The current waveforms show the effectiveness of the transient power sharing

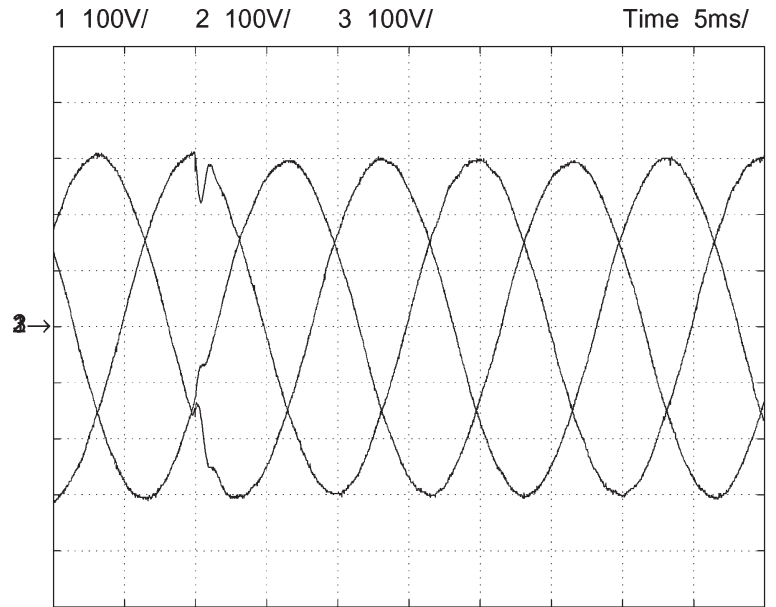

(a)

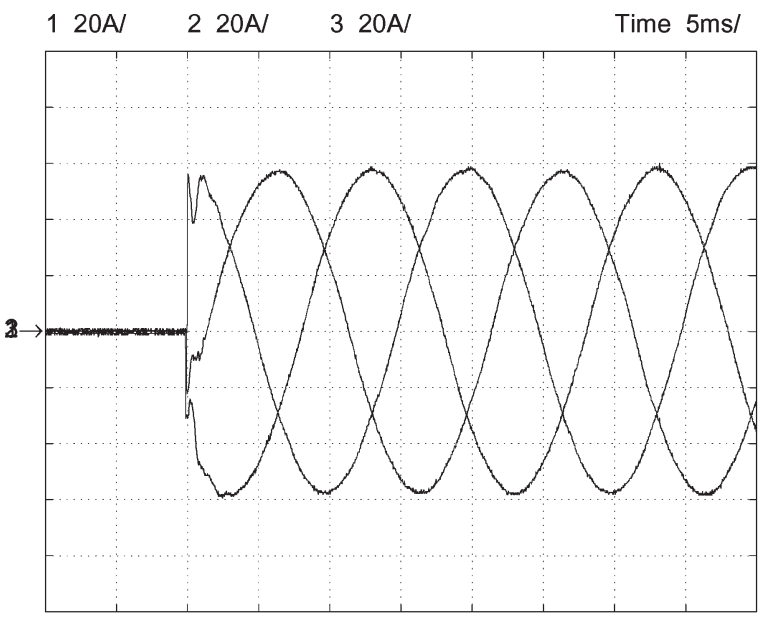

(b)

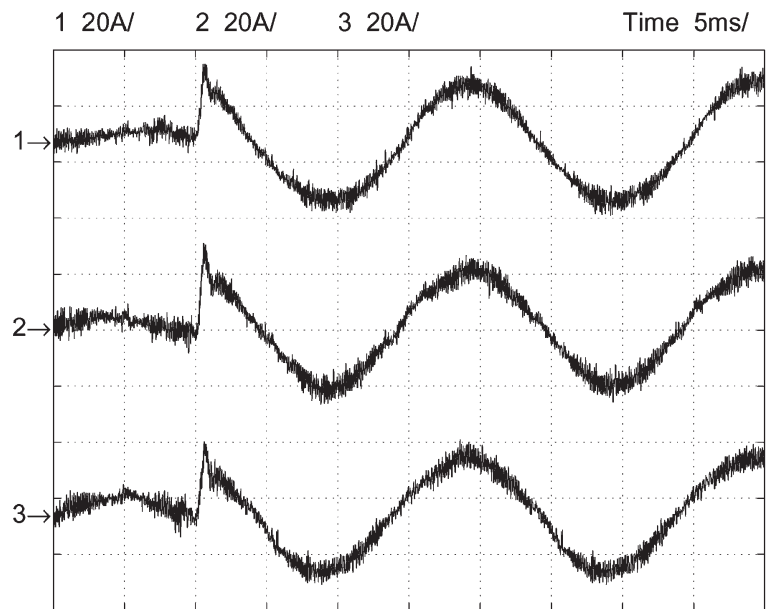

(c)

Fig. 10. Transient of $0 \%-100 \%$ balanced resistive load. (a) Three-phase voltages. (b) Three-phase output currents. (c) Phase- $A$ inductor currents of all inverters.

under distributed control. The transient response is an exacting test because the central and local controller both participate in the response. The currents follow the same wave shape (with very small difference) in response. This result is in contrast to master-slave methods [2], [3], [5] where master takes most of the transient current. In the methods that mimic the operation 

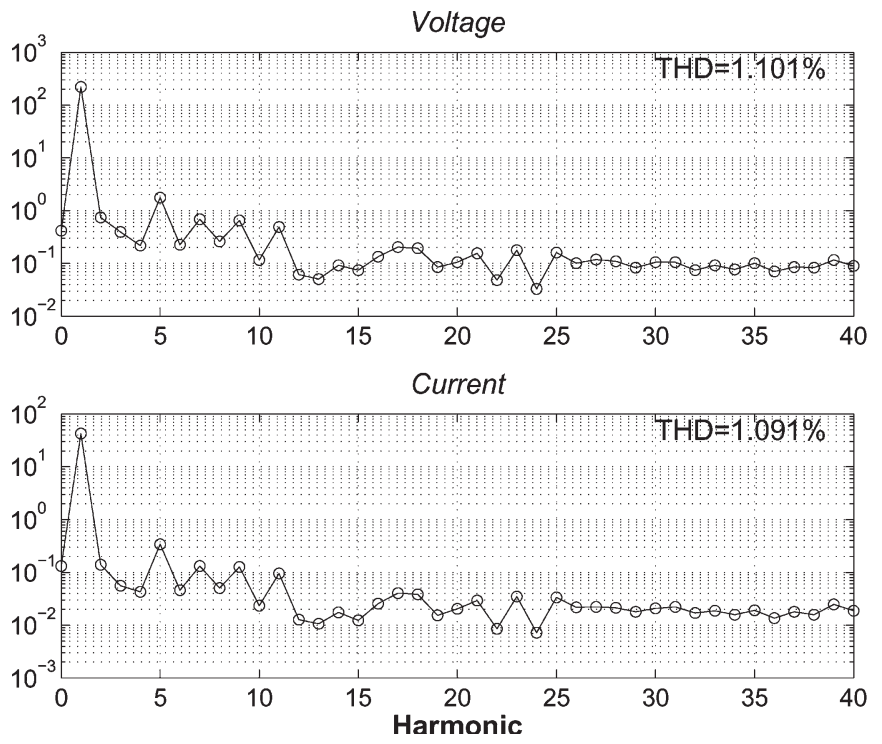

Fig. 11. Spectra of output voltage and current under $100 \%$ balanced resistive load.

of a synchronous generator [6], [13] transient sharing is subject to complex dynamics introduced by the droop (governor) characteristic that was chosen.

Harmonic analysis of phase- $A$ voltage and current is given in Fig. 11. The quality of both the voltage and the current, expressed in terms of the total harmonic distortion (THD), was high and consistent across the three inverters. The pattern of current harmonics is the same as the pattern of voltage harmonics as expected for a resistive load.

The worst case test load for the inverter system in terms of the power quality is a single-phase rectifier load. Its current contains all the odd harmonics and is has negative- and zero-sequence currents of the same magnitude as the positivesequence component. In time domain terms, the load current has a high crest factor. The example used was a rectifier with a large dc-side capacitance and no deliberately introduced ac-side inductance. The dc load resistance was set to consume $25 \%$ of the per-phase power rating of the system.

The waveforms of the phase voltages and the phase- $A$ current are shown in Fig. 12(a). The rms current of phase- $A$ was 16.4 A with the crest factor of 3.4. The phase voltages were $219 \mathrm{~V}_{\mathrm{rms}}, 220 \mathrm{~V}_{\mathrm{rms}}$, and $221 \mathrm{~V}_{\mathrm{rms}}$ with negative- and zerosequence components of $0.9 \%$ and $0.6 \%$, respectively, despite the $100 \%$ components present in the current. The output active and reactive powers in the system and in phase- $A$ were $2.5 \mathrm{~kW}$ and $2.7 \mathrm{kVAr}$.

The spectra of the output voltage and current are shown in Fig. 13. The current contains significant components at all the odd harmonics as expected and the voltage spectrum reveals that the voltage control loop has been largely successful in rejecting these disturbances. The voltage THD was $6.7 \%$ for the phase- $A$. The use of a rotating reference frame in the local controllers means imperfections in the control couple of the other phases so THD levels of $2.4 \%$ and $2.2 \%$ were observed in the other two phases.

The power sharing between units is revealed in Fig. 12(b) which shows the phase- $A$ inductor currents of all three units and

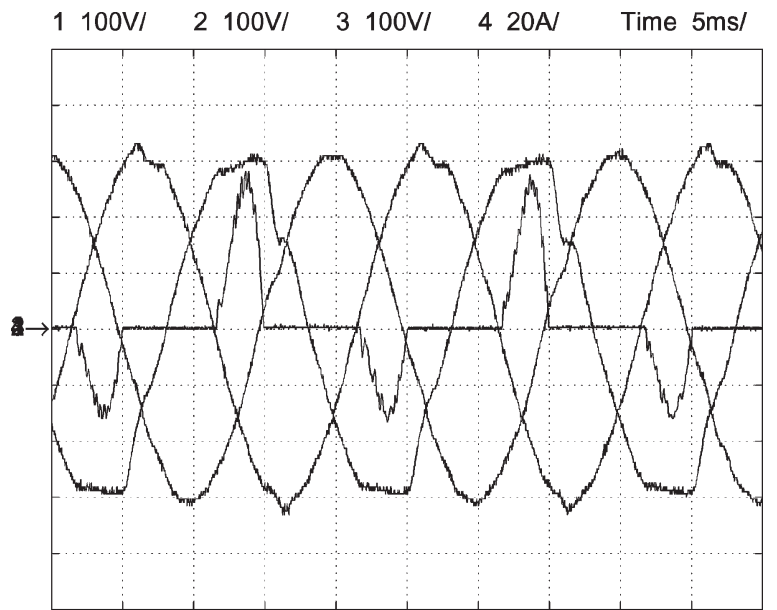

(a)

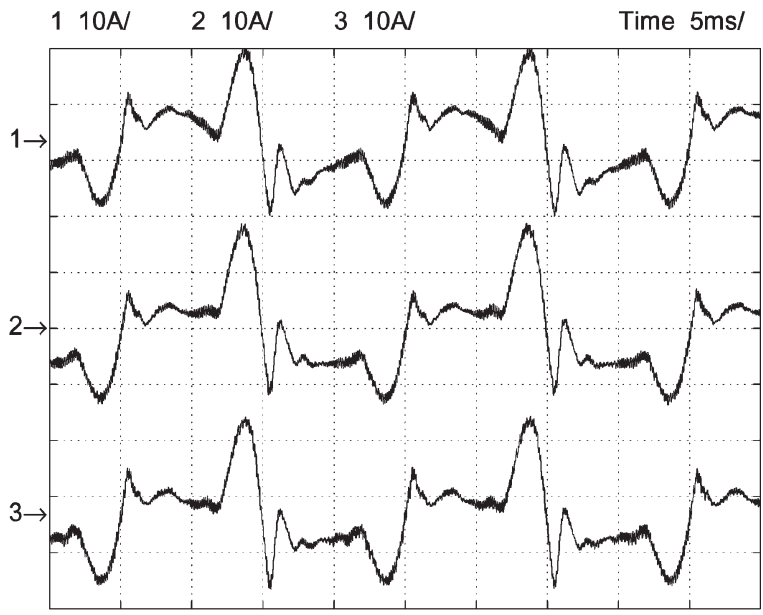

(b)

Fig. 12. Single-phase rectifier load. (a) Three-phase voltages and Phase- $A$ output current. (b) Phase- $A$ inductor currents of all inverters.

the output current drawn from the generator bus. The averaging function of the oscilloscope was used to remove the switching frequency components from the inductor current measurement. The inductor currents in all units followed the same pattern, providing equal sharing of the real power and the harmonic components of the load current.

\section{CONCLUSION}

A scheme for controlling a set of parallel connected inverters in a microgrid has been presented, tested, and compared to conventional control methods. The particular features of the method are that it requires only a low-bandwidth communication link between the units and yet is able to provide good disturbance rejection performance in order to suppress harmonic distortion and unbalance. The method is based on partitioning the control between a low-bandwidth controller that is placed centrally and supplies part of the reference current to the inverters and high-bandwidth controllers distributed locally to each inverter. It has been shown that by using distributed control the disturbance rejection level achieved by a set of inverters separated by communication links can equal that of a single inverter with a single full bandwidth controller. 

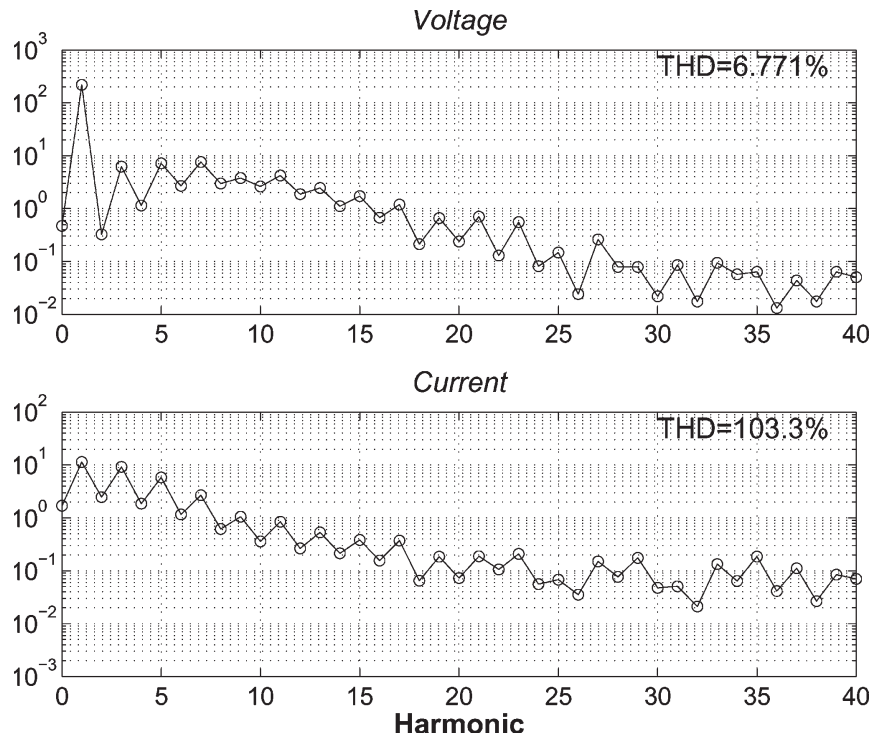

Fig. 13. Spectra of output voltage and current under single-phase rectifier load.

The application of the distributed control to a set of three 10-kVA inverters in a power park microgrid has been demonstrated with experimental results. These results show power sharing was achieved in both steady-state and transient (stepped load) conditions. In particular, it is seen that there are no circulating currents flowing between the inverters. Various test loads were used and as a worst case example a single-phase capacitive diode rectifier was applied. It was observed that the harmonic currents were supplied equally by all three inverters and that the phase voltages were maintained close to perfect balance despite the extreme unbalance of the load current.

The control models of the inverters have been presented and the design requirements for the current and voltage controllers have been given. The choice of characteristic for the low-pass pass filter used for the controller partitioning has also been discussed.

The advantage of distributed control compared to the masterslave methods is in even sharing of harmonic and transient power. In the master-slave arrangement, the master unit provides most of the harmonic or transient duty. In comparison to control methods that mimic the operation of synchronous generator, distributed control has the advantage that near perfect voltage balance and low-harmonic distortion can be achieved through active control. Further, the transient response is nonoscillatory and not subject to the poor dynamics of a frequency-droop-type sharing arrangement.

By using distributed control, a parallel connection of inverters can be controlled with the same performance possible with a single inverter. The high level of power quality expected of inverters is therefore realizable in a power park connection.

\section{REFERENCES}

[1] T. Kawabata and S. Higashino, "Parallel operation of voltage source inverters," IEEE Trans. Ind. Appl., vol. 24, no. 2, pp. 281-287, Mar. 1988.

[2] K. Siri, C. Q. Lee, and T. F. Wu, "Current distribution control for parallel connected converters-Part I," IEEE Trans. Aerosp. Electron. Syst., vol. 28, no. 3, pp. 829-840, Jul. 1992.
[3] _ - "Current distribution control for parallel connected convertersPart II," IEEE Trans. Aerosp. Electron. Syst., vol. 28, no. 3, pp. 841-851, Jul. 1992.

[4] C. Lee, S. Kim, C. Kim, S. Hong, J. Yoo, S. Kim, C. Kim, S. Woo, and S. Sun, "Parallel U.P.S. with a instantaneous current sharing control," in Proc. IEEE IECON, 1998, vol. 1, pp. 568-573.

[5] J.-F. Chen, C.-L. Chu, and Y.-C. Liou, "Modular parallel three-phase inverter system," in Proc. ISIE, 1995, pp. 237-242.

[6] M. Chandorkar, D. Divan, and R. Adapa, "Control of parallel connected inverters in stand-alone AC supply systems," IEEE Trans. Ind. Appl., vol. 29, no. 1, pp. 136-143, Jan./Feb. 1993.

[7] J. M. Guerrero, L. G. de Vicua, J. Matas, M. Castilla, and J. Miret, "A wireless controller to enhance dynamic performance of parallel inverters in distributed generation systems," IEEE Trans. Power Electron., vol. 19, no. 5, pp. 1205-1213, Sep. 2004.

[8] A. Arulampalam, M. Barnes, A. Engler, A. Goodwin, and N. Jenkins, "Control of power electronic interfaces in distributed generation microgrids," Int. J. Electron., vol. 91, no. 9, pp. 503-524, Sep. 2004.

[9] S. Ogasawara, J. Takagaki, and H. Akagi, "A novel control scheme of a parallel current-controlled PWM inverter," IEEE Trans. Ind. Appl., vol. 1A-28, no. 5, pp. 1023-1030, Sep. 1992.

[10] IEEE P1547 Std Draft 06 Standard for Distributed Resources Interconnected with Electric Power Systems, IEEE SCC21, 2000.

[11] M. H. Bollen, Understanding Power Quality Problems. Piscataway, NJ: IEEE Press, 2000.

[12] IEEE Industry Applications Society/Power Engineering Society, IEEE Recomended Practises and Requirements for Harmonic Control in Electrical Power Systems, 1992.

[13] A. Tuladhar, H. Jin, T. Unger, and K. Mauch, "Control of parallel inverters in distributed AC power systems with consideration of line impedance effect," IEEE Trans. Ind. Appl., vol. 36, no. 1, pp. 131-138, Jan. 2000.

[14] R. Zhang, D. Boroyevich, V. H. Prasad, H. Mao, F. C. Lee, and S. Dubovsky, "A three-phase inverter with a neutral leg with space vector modulation," in Proc. IEEE APEC, 1997, pp. 857-863.

[15] R. H. Lasseter, "Control of distributed resources," in Proc. Bulk Power Syst. Dynamics and Control IV, 1998, pp. 323-329.

[16] J.-F. Chen, C.-L. Chu, and C. Huang, "Combination voltage-controlled and current-controlled PWM inverters for parallel operation of UPS," in Proc. IEEE IECON, Nov. 1993, vol. 2, pp. 1111-1116.

[17] M. Prodanović, T. Green, and H. Mansir, "A survey of control methods for parallel three-phase inverters connection," in Proc. IEE PEVD, Sep. 2000, pp. 472-477.

[18] T. Kawabata, T. Miyashita, and Y. Yamamoto, "Dead beat control of three phase PWM inverter," IEEE Trans. Power Electron., vol. 5, no. 1, pp. 21-28, Jan. 1990

[19] A. Nabae, S. Ogasawara, and H. Akagi, "A novel control scheme for current-controlled PWM inverters," IEEE Trans. Ind. Appl., vol. IA-22, no. 4, pp. 697-701, Jul. 1986.

[20] M. Prodanović, T. C. Green, and H. Mansir, "Distributed control method," International Patent Application, Pub. WO/2002/005040.

[21] E. Clarke, Circuit Analysis of AC Power Systems, vol. I. Hoboken, NJ: Wiley, 1943.

[22] Y. Ito and S. Kawauchi, "Microprocessor-based robust digital control for UPS with three-phase PWM inverter," IEEE Trans. Power Electron., vol. 10, no. 2, pp. 196-204, Mar. 1995

[23] M. Prodanović and T. Green, "Control and filter design of three-phase inverters for high power quality grid connection," IEEE Trans. Power Electron., vol. 18, no. 1, pp. 373-380, Jan. 2003.

[24] M. Prodanović, "Power quality and control aspects of parallel connected inverters in distributed generation," Ph.D. dissertation, Imperial College, London, U.K., Jan. 2004.

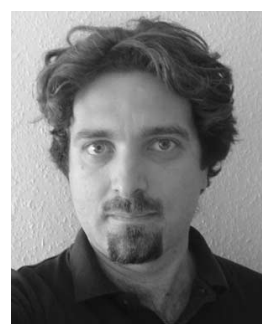

Milan Prodanović (M'01) received the B.Sc. degree in electrical engineering from the University of Belgrade, Serbia, in 1996, and the Ph.D. degree from Imperial College London, London, U.K., in 2004.

$\mathrm{He}$ is currently a Research Associate at Imperial College London. From 1997 to 1999, he was with with GVS Engineering Company, Serbia, developing power electronic circuits and control algorithms for inverter and uninterruptible power system (UPS) systems. His research interests are in digital control of power electronic systems, computer-aided analysis and design of power converters, power quality, microgrids, and distributed generation (DG). 


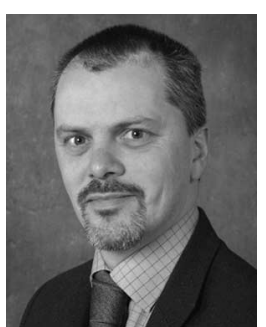

Timothy C. Green (M'88-SM'02) received the B.Sc.(Eng.) degree (first class honors) from Imperial College London, London, U.K., in 1986, and the $\mathrm{Ph} . \mathrm{D}$. degree from Heriot-Watt University, Edinburgh, U.K., in 1990, both in electrical engineering.

He was a Lecturer at Heriot-Watt University until 1994 and is currently a Professor of electrical power engineering at Imperial College London and Deputy Head of the Control and Power Research Group. His research interest is in using power electronics and control to enhance power quality and power delivery. This covers interfaces and controllers for DG, microgrids, active distribution networks, flexible alternating current transmission systems (FACTS), and active power filters. He has an additional line of research in power microelectromechanical systems (MEMS) and energy scavenging.

Dr. Green is a Chartered Engineer in the U.K. and a member of the Institute of Engineering and Technology. 